\title{
Numerical modeling of the strand deposition flow in extrusion-based additive manufacturing
}

\author{
Comminal, Raphaël; Serdeczny, Marcin P.; Pedersen, David B.; Spangenberg, Jon
}

\section{Published in:}

Additive Manufacturing

Link to article, DOI:

10.1016/j.addma.2017.12.013

Publication date:

2018

Document Version

Peer reviewed version

Link back to DTU Orbit

Citation (APA):

Comminal, R., Serdeczny, M. P., Pedersen, D. B., \& Spangenberg, J. (2018). Numerical modeling of the strand deposition flow in extrusion-based additive manufacturing. Additive Manufacturing, 20, 68-76.

https://doi.org/10.1016/i.addma.2017.12.013

\section{General rights}

Copyright and moral rights for the publications made accessible in the public portal are retained by the authors and/or other copyright owners and it is a condition of accessing publications that users recognise and abide by the legal requirements associated with these rights.

- Users may download and print one copy of any publication from the public portal for the purpose of private study or research.

- You may not further distribute the material or use it for any profit-making activity or commercial gain

- You may freely distribute the URL identifying the publication in the public portal 


\section{Accepted Manuscript}

Title: Numerical Modeling of the Strand Deposition Flow in Extrusion-based Additive Manufacturing

Authors: Raphaël Comminal, Marcin P. Serdeczny, David B. Pedersen, Jon Spangenberg

PII: $\quad$ S2214-8604(17)30507-9

DOI: $\quad$ https://doi.org/10.1016/j.addma.2017.12.013

Reference: $\quad$ ADDMA 265

To appear in:

Received date: $\quad 3-11-2017$

Revised date: $\quad$ 6-12-2017

Accepted date: $\quad 30-12-2017$

Please cite this article as: Raphaël Comminal, Marcin P.Serdeczny, David B.Pedersen, Jon Spangenberg, Numerical Modeling of the Strand Deposition Flow in Extrusionbased Additive Manufacturing (2010), https://doi.org/10.1016/j.addma.2017.12.013

This is a PDF file of an unedited manuscript that has been accepted for publication. As a service to our customers we are providing this early version of the manuscript. The manuscript will undergo copyediting, typesetting, and review of the resulting proof before it is published in its final form. Please note that during the production process errors may be discovered which could affect the content, and all legal disclaimers that apply to the journal pertain. 


\title{
Numerical Modeling of the Strand Deposition Flow in Extrusion-based Additive Manufacturing
}

\author{
Raphaël Comminal*, Marcin P. Serdeczny, David B. Pedersen, Jon Spangenberg
}

Department of Mechanical Engineering, Technical University of Denmark, Kgs. Lyngby, Denmark

*Corresponding author: rcom@mek.dtu.dk

Date: 4 Dec. 2017

Revised manuscript submitted to Additive Manufacturing (Ref.: ADDMA_2017_435)

(The changes in the manuscript are highlighted in yellow)

\section{Highlights:}

- Simulations of the material deposition in extrusion-based additive manufacturing

- Prediction of the strand cross-section as function of the processing parameters

- Negative linear relationship between the printing force and the printing speed

\section{Abstract}

We propose a numerical model to simulate the extrusion of a strand of semi-molten material on a moving substrate, within the computation fluid dynamics paradigm. According to the literature, the deposition flow of the strands has an impact on the inter-layer bond formation in extrusion-based additive manufacturing, as well as the surface roughness of the fabricated part. Under the assumptions of an isothermal Newtonian fluid and a creeping laminar flow, the deposition flow is controlled by two parameters: the gap distance between the extrusion nozzle and the substrate, and the velocity ratio of the substrate to the average velocity of the flow inside the nozzle. The numerical simulation fully resolves the deposition flow and provides the cross-section of the printed strand. For the first time, we have quantified the effect of the gap distance and the velocity ratio on the size and the shape of the strand. The cross-section of the strand ranges from being almost cylindrical (for a fast printing and with a large gap) to a flat cuboid with rounded edges (for a slow printing and with a small gap), which substantially differs from the idealized cross-section typically assumed in the literature. Finally, we found that the printing force applied by the extruded material on the substrate has a negative linear relationship with the velocity ratio, for a constant gap.

Keywords: Extrusion-based additive manufacturing; Fused deposition modeling; Numerical simulation; Deposition flow; Strand cross-section; Printing force

\section{Introduction}

Extrusion-based Additive Manufacturing (AM) [1]—commonly known as Fused Deposition Modeling (FDM)is a fabrication technique by which matter is deposited through an extruder nozzle. The part is manufactured layer by layer, by the successive depositions of strands (or threads) on top of a substrate. A detailed review of the extrusion-based AM techniques is provided in [2]. In the case of polymer AM, the extrusion nozzle generally comprises a heated liquefier that melts the feedstock material before the extrusion. The semi-molten material is deposited along a prescribed path that controls the displacement of the extrusion nozzle relative to the substrate. After the extrusion, the strands of the semi-molten material cools down. In the case of polymers, the printed strand welds with the rest of the part via a thermally-driven diffusion process [2]. Extrusion-based AM methods are commonly used for desktop manufacturing of prototypes as well as jigs, fixtures and end-use components for industrial applications. Despite the fact that the extrusion-based AM process is widely employed, limited research has been done on the understanding of the material deposition itself as well as the rheology of polymeric composite materials used as feedstock.

The various processing parameters that influence the physical properties of the printed part, its dimensional accuracy and surface roughness are thoroughly reviewed in $[3,4]$. Several works have been carried out to find empirical relationships between the processing conditions and the final properties of the fabricated parts, using different statistical and heuristic methods, including evolutionary genetic algorithm, fuzzy logic and artificial neural network [4]. These researches aim at identifying the key processing parameters from the experimental data and finding their optimal combinations. Nevertheless, the development of physically-sound models based on deterministic numerical simulations is essential to fully understand the fabrication process and to optimize the design of the machine $[2,5]$. 
Extrusion-based AM is difficult to simulate because it involves multi-physics (thermal and dynamical) phenomena, occurring on different spatial and temporal scales: the material extrusion is a local operation, while the fabrication of an entire part is a global process. Previous modeling works have mostly focused on the thermal and thermo-mechanical behavior of the printed part, after the deposition of the material. Analytical and numerical thermal models have been developed to compute the local temperature history of the printed strand, using lumped capacitance analysis [6-13]. Thermal models have further been coupled to a sintering model [14] (driven by the capillary forces) and healing models $[12,15,16]$ (driven by the intermolecular diffusion), to predict the local bond formation between adjoining strands. Global thermo-mechanical simulations of the entire part have also been utilized to calculate the temperature-induced deformations (shrinkage and warpage) during and after the fabrication [17-22], as well as the residual stresses inside the final part, its degree of crystallinity [20,21], and the potential locations of crack initiation [22]. In these models, the material extrusion is typically emulated by an element activation technique within the thermal/thermo-mechanical finite-element or finite-volume solver. The geometry of the deposited strand is usually simplified to rectangular voxels, in the global simulations, and to a generalized cylinders with circular, elliptic or octagonal cross-sections, in the local models. However, the shape of the strands is an important parameter in the energy balance: the strands exchange heat by conduction through their contact surfaces, while their free surfaces exchange heat by convection and radiation [7,8]. Thus, the accuracy of the thermal models could be enhanced if the geometry of the strands were actually calculated with Computational Fluid Dynamics (CFD) simulations of the deposition flow. In addition, the cross-section of the strands and their overlap interval (necessary for the healing) are key parameters in the analytical model of surface roughness developed by Ahn et al. [23,24]. However, these parameters are rather used as fitting parameters of the model, as they are difficult to estimate [23], without CFD simulations. Moreover, CFD simulations of the strand deposition could be used to predict the interstitial void density between the strands, which enters directly into the models of the mechanical behavior of the fabricated part $[25,26]$.

CFD simulations of non-isothermal flows of power-law fluids inside the extrusion nozzle have been presented in [27-29]. These CFD simulations calculate the flow field inside the extruder, the pressure drop, as well as the melting distance to the entry of the liquefier. Heller et al. [30] used CFD simulations of Newtonian isothermal fluid flows through axisymmetric extrusion nozzles to predict flow-induced fiber orientation of composite polymer inside the nozzle. The effect of the extruder geometry on the flow-induced fiber orientation was investigated; however, their CFD model did not include the deposition flow after the nozzle exit.

McIlroy and Olmsted [31] modelled the disentanglement of an amorphous polymer during the deposition of the material, assuming a smooth ansatz function of the deposition flow which neglects secondary flows. Simplified two-dimensional simulations of the deposition flow have been reported in [27,32]. Dabiri et al. [33] simulated the deposition of multiple strands laid on top of each other, using a high-accuracy three-dimensional surface-tracking algorithm and the Newtonian fluid model. However, the model of Dabiri et al. [33] did not solve the actual extrusion flow, as the material extrusion was emulated by a volumetric source term in the mass conservation equation. To the knowledge of the authors, the three-dimensional extrusion and deposition flow of the semimolten material has only been simulated in the recent work of Du et al. [34], which considers the non-isothermal flows of (non-Newtonian) shear-thinning fluids. In spite of the relatively coarse grid resolution, the CFD simulations of Du et al. [34] were able to predict accurately the area of contact between adjoining strands, for different values of the speed ratio and the layer thickness. Their numerical model has also been used to simulate the deposition flow of a molten metal in fused-coating based AM [35].

The total pressure drop inside the extruder is an important parameter to take into account when designing the liquefier and the feeding system of the printing head [36]. Inadequate operating conditions can cause the buckling of the feedstock filament [37], or the slippage of the filament feedstock on the feeding roller [36,38], which both result in irregularities of the extrusion volumetric flow rate. Bellini et al. [39] proposed a mathematical model that relates the extrusion flow rate to the heat flux of the liquefier and the motorization of the feeding rollers (assuming no buckling and no slippage of the feedstock filament), via the analytical expression of the nonisothermal power-law fluid flow inside an idealized geometry of the extruder consisting of cylindrical and conical sections. Dynamical models of the extruder have also been proposed to detect abnormal flow conditions (e.g. nozzle clogging, overflow, flow disturbances, etc.) from the vibrations of the printing head measured by accelerometers [40,41]. Nevertheless, these dynamical models neglect the printing force that the extruded material applies on the substrate, in spite of the fact that it also contributes to the dynamics of the printing head [40].

In this paper, we present a numerical model of the extrusion of a highly viscous molten material and its deposition onto a moving substrate. This numerical model can be seen as the missing link between the CFD simulations of the internal flow of the molten material inside the extruder, and the thermo-mechanical simulations of the fabricated parts, after the deposition of the strand. For simplicity, we only consider the extrusion of a single strand printed on a planar surface. The simulations predict the cross-section of the strand, as well as the printing force applied by the extruded material on the substrate. We investigate the effects of the velocity ratio of the moving plane to the average velocity inside the nozzle, and the normalized gap between the extruder and the substrate. The remainder of the paper is organized as follows: Section 2 describes the model and numerical methods that we employed. Section 3 discusses the different morphologies of printed strands obtained 
by our CFD simulations, for different processing conditions. Section 4 reports the values of the printing force applied by the extruded material on the substrate. Finally, we conclude with a discussion of the results.

\section{Description of the model}

\subsection{Physical and numerical models}

The model presented in this paper focuses on the extrusion and the deposition of a highly viscous molten material, in the region between the printing head and substrate. The deformation of the molten material is simulated as a fluid flow, within the paradigm of CFD. The dynamics of the fluid flow is governed by the conservation of mass and the Cauchy momentum equation. In addition, we formulate the following assumptions about the physics of the fluid flow:

(i) The material is considered incompressible (with the constant density $\rho$ ), thus the mass conservation translates into the volume conservation.

(ii) Inertial effects are negligible, meaning that we have a creeping flow.

(iii) The material is modeled with a linear constitutive behavior, i.e. a Newtonian fluid, where the internal shear stress $\tau$ is proportional to the shear rate $\dot{\gamma}$ :

$$
\tau=\mu \dot{\gamma}
$$

where $\mu$ is the viscosity of the molten material.

(iv) The liquid adheres to the surfaces of the nozzle and the substrate; there is the no slip condition.

(v) The extrusion process is considered isothermal. In the extrusion-based AM of thermoplastics, the material undergoes a large range of temperatures and is subjected to glass-liquid transition. However, we assume that the variations of temperature within the small gap between the orifice of the extruder and the moving substrate have a negligible effect on the deposition flow. Hence, we have chosen not to include the heating and cooling phenomena in the present model.

Under these assumptions, the flow field is locally governed by the following equations:

$$
\begin{gathered}
\frac{\partial u_{j}}{\partial x_{j}}=0, \\
\rho \frac{\partial u_{i}}{\partial t}=-\frac{\partial p}{\partial x_{i}}+\mu \frac{\partial^{2} u_{i}}{\partial x_{j} \partial x_{j}}+\rho g_{i},
\end{gathered}
$$

where $u_{i}$ is the velocity component in the $i$-direction, $p$ is the local pressure, $g_{i}$ is the gravitational body forces per mass unit in the $i$-direction (i.e. the standard gravity acceleration $g_{0}$ in the vertical direction), $t$ refers to the time, $x_{i}$ to the spatial coordinates, and $j=1,2,3$ is a summation index.

The physics of the extruded material is simulated with a numerical model that includes the exit of the extrusion nozzle and the gap between the printing head and substrate. In the present model, the position of the nozzle is fixed, while the substrate moves with a controlled speed. However, since the movements of the printing head and substrate are relative to each other, and the accelerations are neglected, the model equally covers the opposite configuration where the printing head moves and the substrate is fixed.

The geometry of the numerical model is represented in Fig. 1. The extrusion nozzle is a cylindrical tube with the inner diameter $D$ and the outer diameter $2 D$. The numerical model assumes that the flow at the nozzle exit is laminar and fully-developed. Consequently, the length of the nozzle are unimportant for the flow exiting the nozzle. The fully-developed flow inside the nozzle has an axisymmetric parabolic profile:

$$
u r=2 U\left(1-\frac{r^{2}}{D / 2^{2}}\right), \text { for } 0 \leq r \leq D / 2,
$$

where $U$ is the average velocity inside the nozzle, which is directly linked to the speed $V_{f}$ and diameter $D_{f}$ of the plastic filament feeding the printing head:

$$
U=V_{f}\left(\frac{D_{f}}{D}\right)^{2} .
$$

Below the printing head, the moving substrate is modeled by a perpendicular plane, with a gap distance $g$ from the nozzle exit. The exterior of the printing nozzle includes a rectangular domain. By virtue of symmetry, the numerical model only includes half of the three-dimensional geometry. The remaining external surfaces of the numerical model are outlet boundaries, where the material is free to exit. All the dimensions of the numerical 
model are normalized by the nozzle diameter $D$, while the velocity $V$ of the moving substrate is normalized by the average velocity inside the nozzle. The fluid flow of the extruded material is simulated for different printing conditions. The numerical values chosen for the numerical simulations are provided in Table 1. Gravity is included in the simulations; however, its effect on the fluid flow is negligible.

Under the assumptions of a creeping flow and a Newtonian fluid, the viscosity of the molten material only affects the pressure gradient inside the nozzle, but it does not change the dynamics of the material deposition. Therefore, the actual values of the density and viscosity are not important, as long as the Reynolds number $R e=\rho U D / \mu$ is sufficiently low (below one). Thus, the printing conditions of the strand are fully characterized by two dimensionless parameters: the velocity ratio $V / U$, and the normalized gap $g / D$. Hence, the predictions of the numerical simulations apply to any molten material having a high viscosity within the operating conditions of the extruder, which is the case for most thermoplastics used in 3D printing, such as PLA and ABS. Twelve different combinations of $V / U$ and $g / D$ have been tested in the numerical simulations.

\subsection{Principle of the numerical simulation}

The fluid flow is simulated with the software ANSYS ${ }^{\circledR}$ Fluent R15.0 [42], which is based on the finite-volume method. The governing equations (2) and (3) are integrated over a finite set of tetrahedral control volumes that meshes the simulation domain. Details of the tetrahedral mesh are represented in Fig. 2. Following a cell-centered discretization, the numerical solver computes discrete values of the continuous velocity and pressure fields at the center of the control volumes. The values at any other locations are interpolated from the discrete values, whenever required. The numerical scheme evaluates the advection and diffusion fluxes of momentum through all the faces of the control volumes. Then, the accumulated quantity of momentum inside each control volume is updated according to its net fluxes [43]. As the fluid is incompressible, the pressure field is a result of the continuity constraint. A pressure equation is derived from the mass conservations. Moreover, the free surface of the extruded material is captured by an algebraic coupled level-set/volume-of-fluid method, which computes the transport of two additional field variables tracking the position of the surface. We refer the readers to the following papers [44-47] for a review of the volume-of-fluid method, the level-set method, and the coupled levelset/volume-of-fluid method.

The evolution of the system is solved incrementally, at regular time intervals. The precision of the numerical simulation depends on the size of the control volumes and time-step intervals. In our simulations, the maximum size of the control volumes is $\delta l=0.06 \mathrm{~mm}$, and the time-step interval is $\delta t=0.01 \mathrm{sec}$. The discretized momentum equations and pressure equations relative to the set of control volumes are solved with an implicit solver that guaranty the stability of the numerical scheme. At each incremental time step, the numerical algorithm computes the new values of the primary discrete variables that are the local velocities, the pressure and the position of the free surface of the extruded material. Secondary results, such as the streamline of the flow, the shear rate, the viscous stress, or the printing force applied by the extruded material on the substrate, are computed from the primary variables. Fig. 3 represents examples of numerical results that can be visualized after the simulation is completed, for instance the streamlines of the flow and the free surface of the printed strand.

\section{Morphology of the printed strand}

The shape of the printed strand is captured by the free surface-tracking algorithm. Fig. 4 represents examples of side- and top-views of material deposition, for two printing conditions: (a) a fast printing with a large gap, and (b) a slow printing with a small gap. We can see that the extruded material spreads more on the substrate, at the front and on the side of the printing head, for a slow printing speed and with a small gap. In contrast, the extruded material has less side flow and tends to stay behind of the printing head, for a fast printing and with a large gap. After the extrusion, the printed strand quickly reaches a quiescent state. By virtue of the volume conservation, the volumetric flow rate of the material deposited on the moving substrate is the same as the volumetric flow rate in the nozzle. Therefore, the area $\mathcal{A}$ of the cross-section of the strand is given by the analytical expression:

$$
\mathcal{A}=\frac{\pi D^{2} U}{4 V} \text {. }
$$

It is interesting to note that the area of the strand cross-section does not depend on the gap distance. However, both the gap distance and velocity ratio influence the shape of the strand cross-section. 
Fig. 5 shows the variety of all the strand cross-sections that have been obtained within the numerical simulations, for different gaps and velocity ratios. The shape of the printed strand ranges from being almost cylindrical (for a fast printing and with a large gap) to a flat cuboid with rounded edges (for a slow printing and with a small gap). Both the reduction of the gap and the reduction of the printing speed enhance the side flow of the extruded material, which flattens the shape of the printed strand.

The normalized strand thickness $H / D$ and the normalized strand width $W / D$ are reported in Fig. 6. It is often assumed in the literature that the strand thickness equals the gap; however, our numerical results show that the strand thickness vary between $-10 \%$ to $+35 \%$ of the value of gap, in the range of the processing parameters that we have investigated. As the extruded material adheres both to the moving substrate and to the static cap of the extruder, it is subjected to shear deformation, within the gap region. After the material passes through the gap region, the shear flow gets rearranged into a uniform flow with velocity $V$, which can yield the shrinkage of the extruded material, by virtue of mass conservation. We can see in Fig. 6(left) that the thickness of the strand shrinks below the value of the gap distance when the printing speed or the gap distance are high. Moreover, in the case of a slow printing or a small gap, the extruded material flows beside the cap of extruder (because of a radial pressure difference), as seen in Fig. 4(b). As a consequence, the side flow of the extruded material is not restrained anymore by the cap of the extruder, which explains that the strand thickness exceeds the gap distance. In addition, the upper surface of the printed strand become concave, as it can be seen in Fig. 5. This phenomenon has also been reported in [48]. Furthermore, we must note that the idealized linear relation $H=g$ is only valid when

$g<D \sqrt{U / V}$. For a gap value beyond that limit (not covered by our numerical simulations), the material would be extruded without contact with the cap of the extruder, leading to a smooth deposition on the substrate without secondary flows (like in the model presented in [31]). In that case, the strand thickness would become independent of the gap distance.

The strand width, plotted in Fig. 6(right), is compared with the width of the idealized cross-sections drawn in Fig. 7, which are often assumed in the literature. The dotted lines (…) in Fig. 6(right) are for the ellipse crosssections, the dash-dot lines (-- ) are for the oblong cross-sections, the dashed lines (- - ) are for the cuboid crosssections, while the solid lines with markers represent the results of the numerical simulations. We can see that the strand width calculated with the numerical model substantially deviates from the widths of the idealized crosssections.

The aspect ratio $W / H$ and the compactness $\mathcal{A} / W H$ of the cross-section are also reported in Fig. 8. Again, we can see that the aspect ratio and the compactness of the printed strand differ from the idealized values. The aspect ratio of the strand globally increases as the printing speed and the gap distance are reduced. The compactness of the strand cross-section does not present a clear pattern, as it is affected by the side flow beside the extruder cap. Moreover, it is expected that the outer dimensions of the cap of the extruder (not investigated here) influence the aspect ratio and compactness of the cross-section.

\section{Printing force}

The viscous flow develops a pressure gradient along the extrusion nozzle, to overcome the flow resistance coming from the adherence of the molten material to the nozzle wall. The pressure gradient $P_{z}$ driving the laminar creeping flow inside the nozzle is given by the Hagen-Poiseuille law:

$$
P_{z}=\frac{32 \mu U}{D^{2}} \text {. }
$$

The extruded material applies a printing force $F$ on the substrate, which corresponds to the integral of the pressure field (minus a gauge pressure $P_{\text {atm }}$ ) over the surface of the substrate:

$$
F=\int_{\text {Substrate }} P-P_{\text {atm }} d S .
$$

The value of the pressure field is normalized by the average viscous stress $\tau_{a}$ inside the nozzle:

$$
\bar{P}=P-P_{\text {atm }} / \tau_{a} \text {, }
$$

where

$$
\tau_{a}=\mu \dot{\gamma}_{a}
$$

and $\dot{\gamma}_{a}$ is the average shear-rate of the fully-developed laminar pipe flow:

$$
\dot{\gamma}_{a}=\frac{4}{\pi D^{2}} \int_{0}^{2 \pi} \int_{0}^{\frac{D}{2}} \dot{\gamma} r r d r d \theta=\frac{16 U}{3 D} .
$$

The distribution of the normalized pressure on the substrate is represented in Fig. 9, for different values of $g / D$ and $V / U$. As expected, the extruded material applies a larger pressure on the substrate when the gap 
distance is small and when the printing velocity is reduced. For the printing conditions with a small gap or a low printing speed, the extruded material applies a pressure field almost axisymmetric, with the maximum pressure just below the center of the nozzle, as illustrated in Fig. 9(top). For larger gaps or faster printing speeds, the pressure level drops, but an excess of pressure appears at the front of the printing head, as seen in Fig. 9(middle). Finally, the pressure excess at the front of the printing head disappears when the substrate velocity or the gap is further increased, and the maximum pressure becomes situated below the printing nozzle again; see Fig. 9(bottom).

The normalized printing force $F / F_{0}$ is calculated via the numerical integration of the normalized pressure distribution over the substrate:

$$
\frac{F}{F_{0}}=\frac{4}{\pi D^{2}} \int_{\text {Substrate }} \bar{P} d S
$$

where the reference force

$$
F_{0}=\frac{1}{4} \pi D^{2} \tau_{a}
$$

is the total internal viscous force of the material, inside a cross-section of the extrusion nozzle. The normalized printing forces for all the printing parameters that we have simulated are reported in Fig. 10. As expected, the printing force increases as the gap and the substrate velocity are reduced. Moreover, at a constant gap, the variation of the printing force with respect to the printing speed follows a negative linear relationship, for the range of the processing parameters that we investigated.

\section{Conclusions}

We have presented a numerical model within the paradigm of CFD to simulate the deposition of a highly viscous molten material on a moving plane, in extrusion-based AM. The CFD simulations fully resolves the velocity- and pressure-fields based on the mass conservation and the momentum balance inside the extruded material, while the free surface of the printed strand is tracked by a surface-capturing algorithm. Under the assumptions of an incompressible creeping flow and the first order approximation of the constitutive law as a Newtonian fluid without temperature-dependency of the material properties, the hydrodynamics of the extruded material is solely parametrized by two processing variables: the normalized gap distance, and the ratio of substrate velocity to the average velocity of the flow inside the nozzle. The potential effects of non-linearities and temperature-dependences in the constitutive law are neglected. However, the predictions of the current model are quite general and apply to many of the molten material used in extrusion-based AM.

According to the literature, the shape of the printed strand has a direct impact on the surface roughness of the manufactured part, the cooling process after the printing, as well as the bond formation between the adjoining strands and the overlaid layers. Hence, it influences indirectly the dimensional accuracy (via the thermally induced deformations), and the mechanical properties of the final part (via the bond formation), although they depend also on other processing parameters such as the extrusion temperatures, the envelope temperatures, and the tool path strategy.

For the first time, we have quantified the effects of the velocity ratio and the gap on the shape and the dimensions of the cross-section of the printed strand, as well as the printing force applied on the substrate. The shape of the strand ranges from being almost cylindrical (for a fast printing and with a large gap) to a flat cuboid with rounded edges (for a slow printing and with a small gap). At a constant gap, the printing force appears to decrease linearly with increasing printing speeds, under the processing conditions that we investigated. An increase in the gap increases the thickness of the strand, but decreases its width and its aspect ratio. Moreover, both the strand width, the strand thickness, as well as the aspect ratio of the strand cross-section decrease when the printing speed increases. The compactness of the strand cross-section presents non-monotonic variations, with a maximum value of around $90 \%$. The outer dimensions of the cap of the extruder also have an influence on the side flow of the extruded material, when the gap distance or the printing speed is reduced. Moreover, the dimensions of the printed strand calculated with the CFD model substantially deviate from the idealized dimensions of the elliptic, oblong and cuboid cross-sections. Both the dimensions and the compactness of the strand are crucial variables to take into account for the tool path planning. Nevertheless, many tool path planning algorithms relies on idealized layer thickness and strand width, which could be a cause of geometrical errors in extrusion-based AM.

The deposition of multiple strand layers will be simulated in future works, to predict the void density of the fabricated part as a function of the processing parameters. The CFD model could also test the effect of different nozzle geometries. Finally, real-time measurements of the printing force could also be used to control the flow conditions in the nozzle, as it is has a negative linear relationship with the inverse of the flow rate, for a steady printing speed and a constant gap.

\section{Acknowledgement}


The authors would like to acknowledge the support of the Danish Council for Independent Research (DFF) | Technology and Production Sciences (FTP) (Contract No. 7017-00128). 


\section{References}

[1] ISO/ASTM 52900, First edition, 2015-12-15.

[2] B.N. Turner, R. Strong, S.A. Gold, A review of melt extrusion additive manufacturing processes: I. Process design and modeling, Rapid Prototyping Journal 20 (2014) 192-204.

[3] B.N. Turner, S.A. Gold, A review of melt extrusion additive manufacturing processes: II. Materials, dimensional accuracy, and surface roughness, Rapid Prototyping Journal 21 (2015) 250-261.

[4] O.A. Mohamed, S.H. Masood, J.L. Bhowmik, Optimization of fused deposition modeling process parameters: a review of current research and future prospects, Advances in Manufacturing 3 (2015) 42-53.

[5] H. Bikas, P. Stavropoulos, G. Chryssolouris, Additive manufacturing methods and modelling approaches: a critical review, The International Journal of Advanced Manufacturing Technology 83 (2016) 389-405.

[6] M.A. Yardimci, S.I. Guceri, M.K. Agarwala, S.C. Danforth, Part quality prediction tools for fused deposition processing, Proceedings of Solid Freeform Fabrication Symposium, University of Texas at Austin, 1996, pp. 539-548.

[7] S.F. Costa, F.M. Duarte, J.A. Covas, Towards modelling of Free Form Extrusion: analytical solution of transient heat transfer, International Journal of Material Forming 1 (2008) 703-706.

[8] S.F. Costa, F.M. Duarte, J.A. Covas, Thermal conditions affecting heat transfer in FDM/FFE: a contribution towards the numerical modelling of the process, Virtual and Physical Prototyping 10 (2015) 35-46.

[9] Y. Zhou, T. Nyberg, G. Xiong, D. Liu, Temperature analysis in the fused deposition modeling process, in 3rd International Conference on Information Science and Control Engineering (ICISCE), 2016, pp. 678-682.

[10] M.L. Jensen, M.R. Sonne, J.H. Hattel, H.N. Hansen, Thermal modelling of extrusion based additive manufacturing of composite materials, in 21st International Conference on Composite Materials (ICCM-21), 2017.

[11] J. Zhang, X.Z. Wang, W.W. Yu, Y.H. Deng, Numerical investigation of the influence of process conditions on the temperature variation in fused deposition modeling, Materials \& Design 130 (2017) 59-68.

[12] S.F. Costa, F.M. Duarte, J.A. Covas, Estimation of filament temperature and adhesion development in fused deposition techniques, Journal of Materials Processing Technology 245 (2017) 167-179.

[13] Y. Zhang, V. Shapiro, Linear-Time Thermal Simulation of As-Manufactured FDM Components, University of Wisconsin-Madison, 2017.

[14] C. Bellehumeur, L. Li, Q. Sun, P. Gu, Modeling of bond formation between polymer filaments in the fused deposition modeling process, Journal of Manufacturing Processes 6 (2004) 170-178.

[15] J.P. Thomas, J.F. Rodríguez, Modeling the fracture strength between fused deposition extruded roads, Proceedings of the 11th Solid Freeform Fabrication Symposium, University of Texas at Austin, 2000, pp. 1623.

[16] C. McIlroy, P.D. Olmsted, Disentanglement Effects on the Welding Behaviour of Polymer Melts during the Fused-Filament-Fabrication Method for Additive Manufacturing, arXiv preprint 1703.09295v1, 2017.

[17] Y. Zhang, Y.K. Chou, Three-dimensional finite element analysis simulations of the fused deposition modelling process, Proceedings of the Institution of Mechanical Engineers, Part B: Journal of Engineering Manufacture 220 (2006) 1663-1671.

[18] Y. Zhang, K. Chou, A parametric study of part distortions in fused deposition modelling using threedimensional finite element analysis, Proceedings of the Institution of Mechanical Engineers, Part B: Journal of Engineering Manufacture 222 (2008) 959-968.

[19] X. Zhou, S.J. Hsieh, Y. Sun, Experimental and numerical investigation of the thermal behaviour of polylactic acid during the fused deposition process, Virtual and Physical Prototyping 12 (2017) 221-233.

[20] B. Brenken, E. Barocio, A.J. Favaloro, R.B. Pipes, Simulation of Semi-Crystalline Composites in the Extrusion Deposition Additive Manufacturing Process, Science in the Age of Experience (2017) 90-102.

[21] A.J. Favaloro, B. Brenken, E. Barocio, R.B. Pipes, Simulation of Polymeric Composites Additive Manufacturing using Abaqus, Science in the Age of Experience (2017) 103-114.

[22] M.R. Talagani, S. DorMohammadi, R. Dutton, C. Godines, H. Baid, F. Abdi, V. Kunc, B. Compton, S. Simunovic, C. Duty, L. Love, B. Post, C. Blue, Numerical simulation of big area additive manufacturing (3D printing) of a full size car, SAMPE Journal 51 (2015) 27-36.

[23] D. Ahn, J.H. Kweon, S. Kwon, J. Song, S. Lee, Representation of surface roughness in fused deposition modeling, Journal of Materials Processing Technology 209 (2009) 5593-5600.

[24] D. Ahn, J.H. Kweon, J.H. Choi, S.H. Lee, Relation between surface roughness and overlap interval in fused deposition modeling, Advanced Materials Research 264 (2011) 1625-1630.

[25] L. Li, Q. Sun, C. Bellehumeur, P. Gu, Composite modeling and analysis for fabrication of FDM prototypes with locally controlled properties, Journal of Manufacturing Processes 4 (2002) 129-141.

[26] J.F. Rodríguez, J.P. Thomas, J.E. Renaud, Mechanical behavior of acrylonitrile butadiene styrene fused deposition materials modeling, Rapid Prototyping Journal 9 (2003) 219-230.

[27] A. Bellini, Fused deposition of ceramics: a comprehensive experimental, analytical and computational study of material behavior, fabrication process and equipment design, Ph.D. thesis, Drexel University, 2002. 
[28] H.S. Ramanath, C.K. Chua, K.F. Leong, K.D. Shah, Melt flow behaviour of poly-e-caprolactone in fused deposition modelling, Journal of Materials Science: Materials in Medicine 19 (2008) 2541-2550.

[29] N. Mostafa, H.M. Syed, S. Igor, G. Andrew, A study of melt flow analysis of an ABS-Iron composite in fused deposition modelling process, Tsinghua Science \& Technology 14 (2009) 29-37.

[30] B.P. Heller, D.E. Smith, D.A. Jack, Effects of extrudate swell and nozzle geometry on fiber orientation in Fused Filament Fabrication nozzle flow, Additive Manufacturing 12 (2016) 252-264.

[31] C. McIlroy, P.D. Olmsted, Deformation of an amorphous polymer during the fused-filament-fabrication method for additive manufacturing, Journal of Rheology 61 (2017) 379-397.

[32] R. Comminal, J.H. Hattel, J. Spangenberg, Numerical Simulations of Planar Extrusion and Fused Filament Fabrication of Non-Newtonian Fluids, Annual Transactions of the Nordic Rheology Society 25 (2017) 263270.

[33] S. Dabiri, S. Schmid, G. Tryggvason, Fully Resolved Numerical Simulations of Fused Deposition Modeling, Proceedings of the ASME 2014 International Manufacturing Science and Engineering Conference MSEC2014, 2014.

[34] J. Du, Z. Wei, X. Wang, J. Wang, Z. Chen, An improved fused deposition modeling process for forming large-size thin-walled parts, Journal of Materials Processing Technology 234 (2016) 332-341.

[35] X. Fang, J. Du, Z. Wei, X. Wang, P. He, H. Bai, B. Wang, J. Chen, R. Geng, B. Lu, Study on Metal Deposit in the Fused-coating Based Additive Manufacturing, Procedia CIRP 55 (2016) 115-121.

[36] M.E. Mackay, Z.R. Swain, C.R. Banbury, D.D. Phan, D.A. Edwards, The performance of the hot end in a plasticating 3D printer, Journal of Rheology 61 (2017) 229-236.

[37] N. Venkataraman, S. Rangarajan, M.J. Matthewson, A. Safari, S.C. Danforth, A. Yardimci, S.I. Guceri, Mechanical and rheological properties of feedstock material for fused deposition of ceramics and metals (FDC and FDMet) and their relationship to process performance, Proceedings of Solid Freeform Fabrication Symposium, University of Texas at Austin, 1999, pp. 351-360.

[38] K.Y. Jiang, Y.H. Gu, Controlling parameters for polymer melting and extrusion in FDM, Key Engineering Materials 259 (2004) 667-671.

[39] A. Bellini, S. Güçeri, M. Bertoldi, Liquefier dynamics in fused deposition, Journal of Manufacturing Science and Engineering 126 (2004) 237-246.

[40] S. Bukkapatnam, N. Clark, Dynamic modeling and monitoring of contour crafting-An extrusion-based layered manufacturing process, Journal of Manufacturing Science and Engineering 129 (2007) 135-142.

[41] Y. Tlegenov, Y.S. Wong, G.S. Hong, A dynamic model for nozzle clog monitoring in fused deposition modelling, Rapid Prototyping Journal 23 (2017) 391-400.

[42] ANSYS® Fluent, Release 15.0, 2013, ANSYS Fluent Theory Guide, ANSYS Inc., Canonsburg, PA, USA.

[43] S. Patankar, Numerical heat transfer and fluid flow, CRC press, 1980.

[44] R. Scardovelli, S. Zaleski, Direct numerical simulation of free-surface and interfacial flow, Annual Review of Fluid Mechanics 31 (1999) 567-603.

[45] R. Comminal, J. Spangenberg, J.H. Hattel, Cellwise conservative unsplit advection for the volume of fluid method, Journal of Computational Physics 283 (2015) 582-608.

[46] J.A. Sethian, P. Smereka, Level set methods for fluid interfaces, Annual Review of Fluid Mechanics 35 (2003) 341-372.

[47] M. Sussman, E.G. Puckett, A coupled level set and volume-of-fluid method for computing 3D and axisymmetric incompressible two-phase flows, Journal of Computational Physics 162 (2000) 301-337.

[48] W. Li, A. Ghazanfari, M.C. Leu, R.G. Landers, Extrusion-on-demand methods for high solids loading ceramic paste in freeform extrusion fabrication, Virtual and Physical Prototyping 12 (2017) 193-205. 


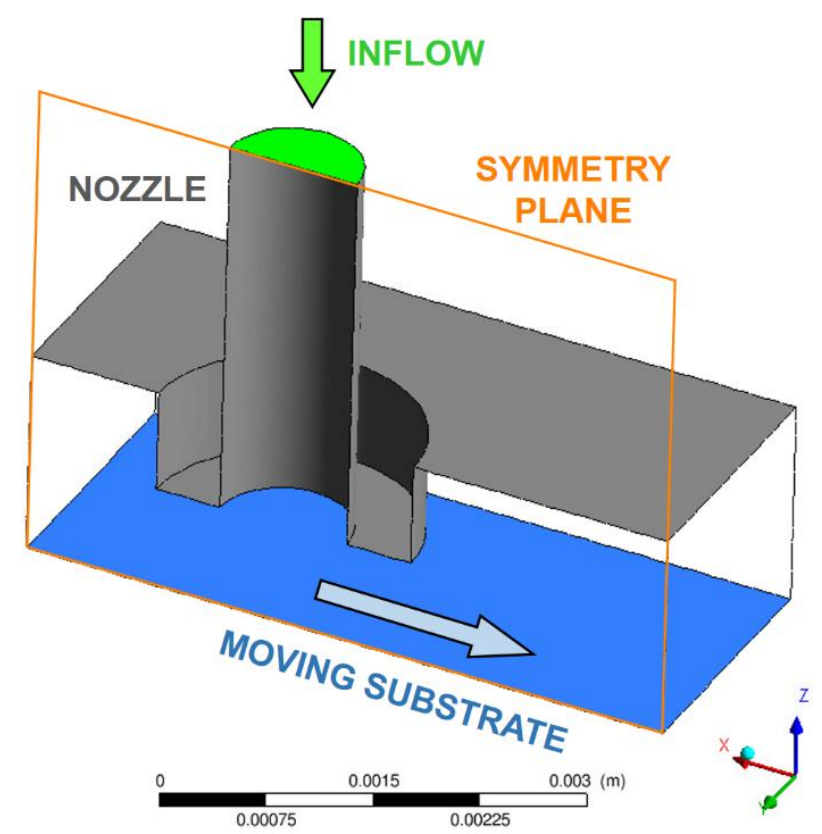

Figure 1. Geometry of the numerical model. 


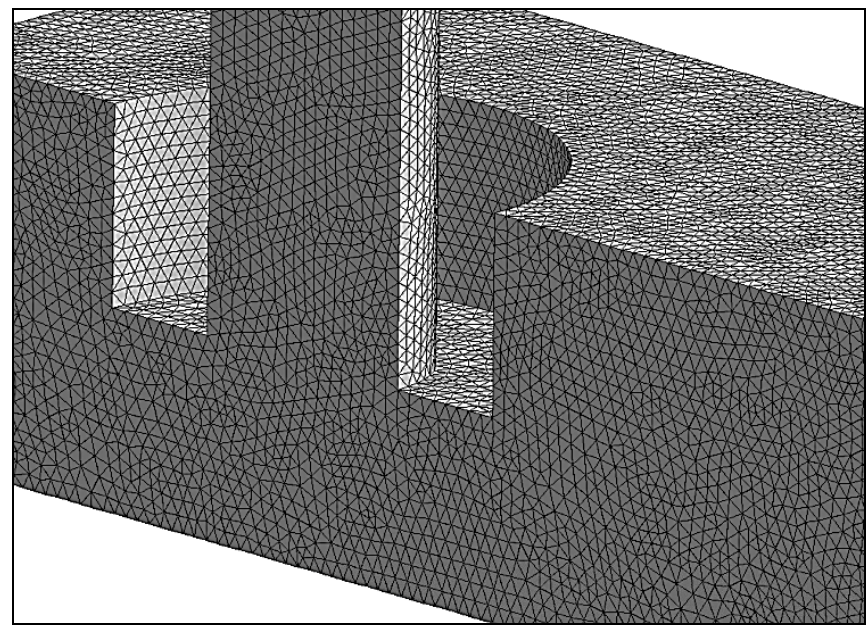

Figure 2. Details of the tetrahedral mesh. 

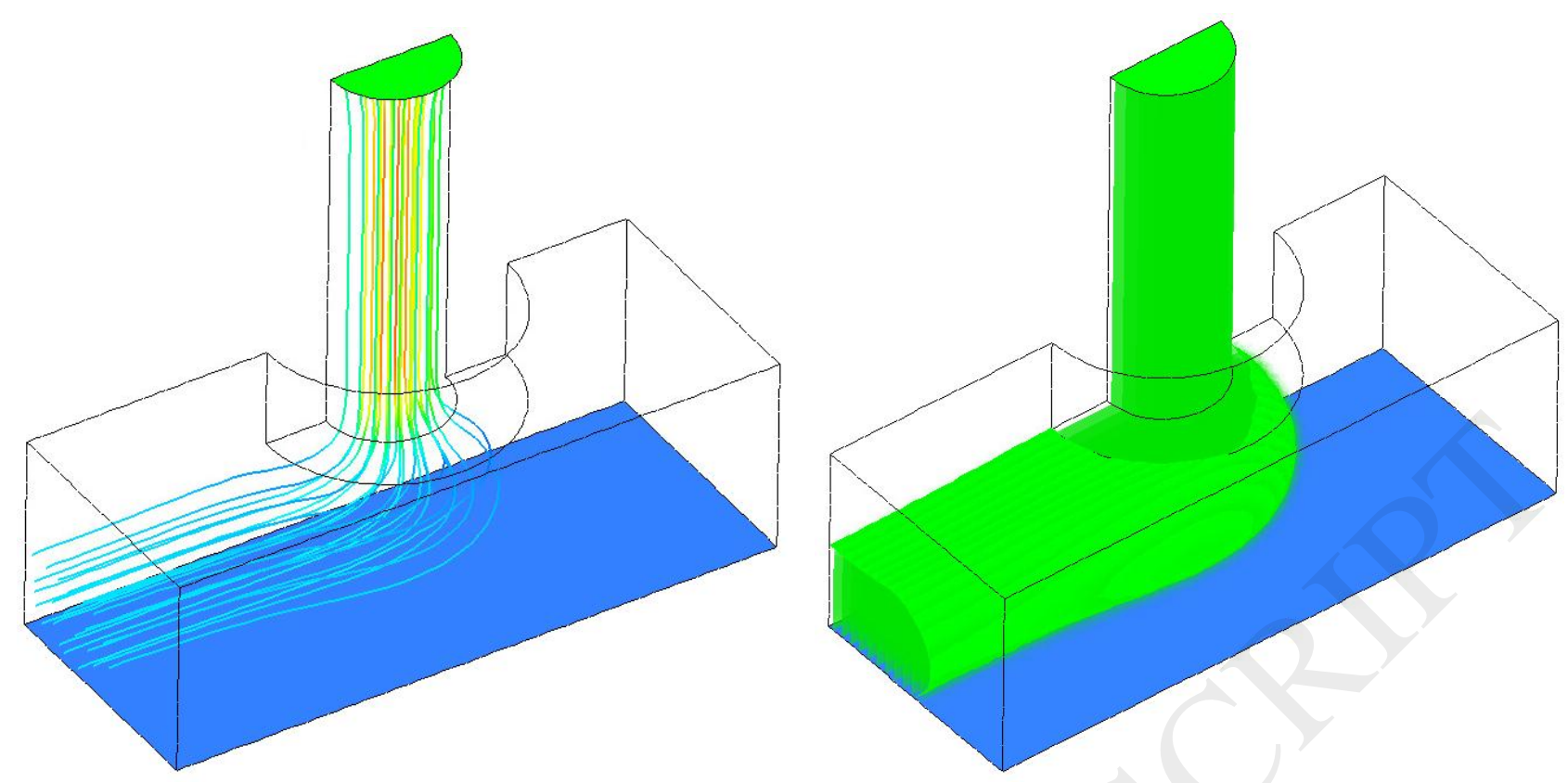

Figure 3. Examples of simulation results with the numerical model: streamlines of the extruded material (left), and free surface of the printed strand (right), for $g / D=0.8$ and $V / U=0.5$. 
(a) $g / D=1.0$ $V / U=1.0$ (b) $g / D=0.4$ $V / U=0.5$

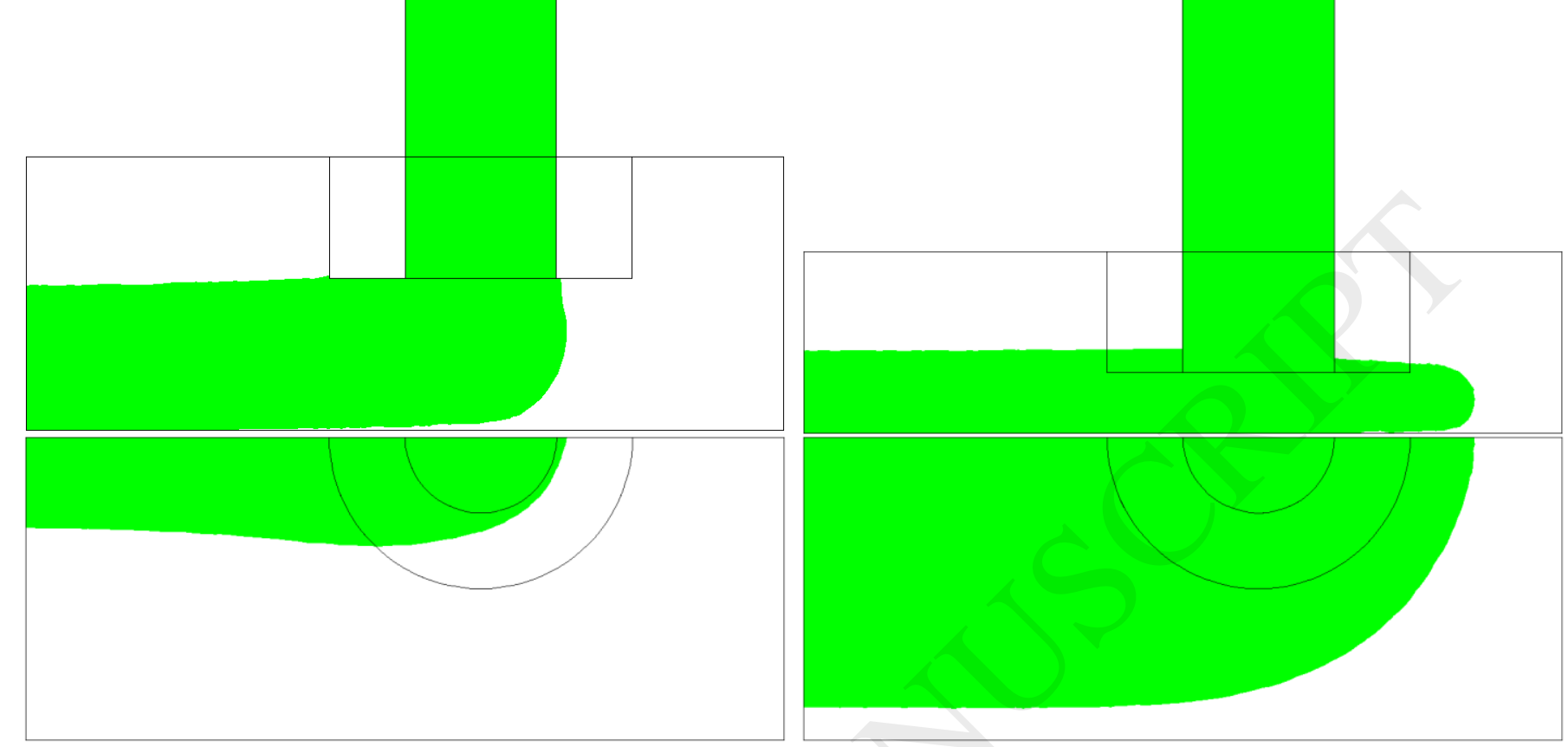

Figure 4. Side- and top-views of the extruded material: (a) for $g / D=1.0$ and $V / U=1.0$, (b) for $g / D=0.4$ and $V / U=0.5$. 

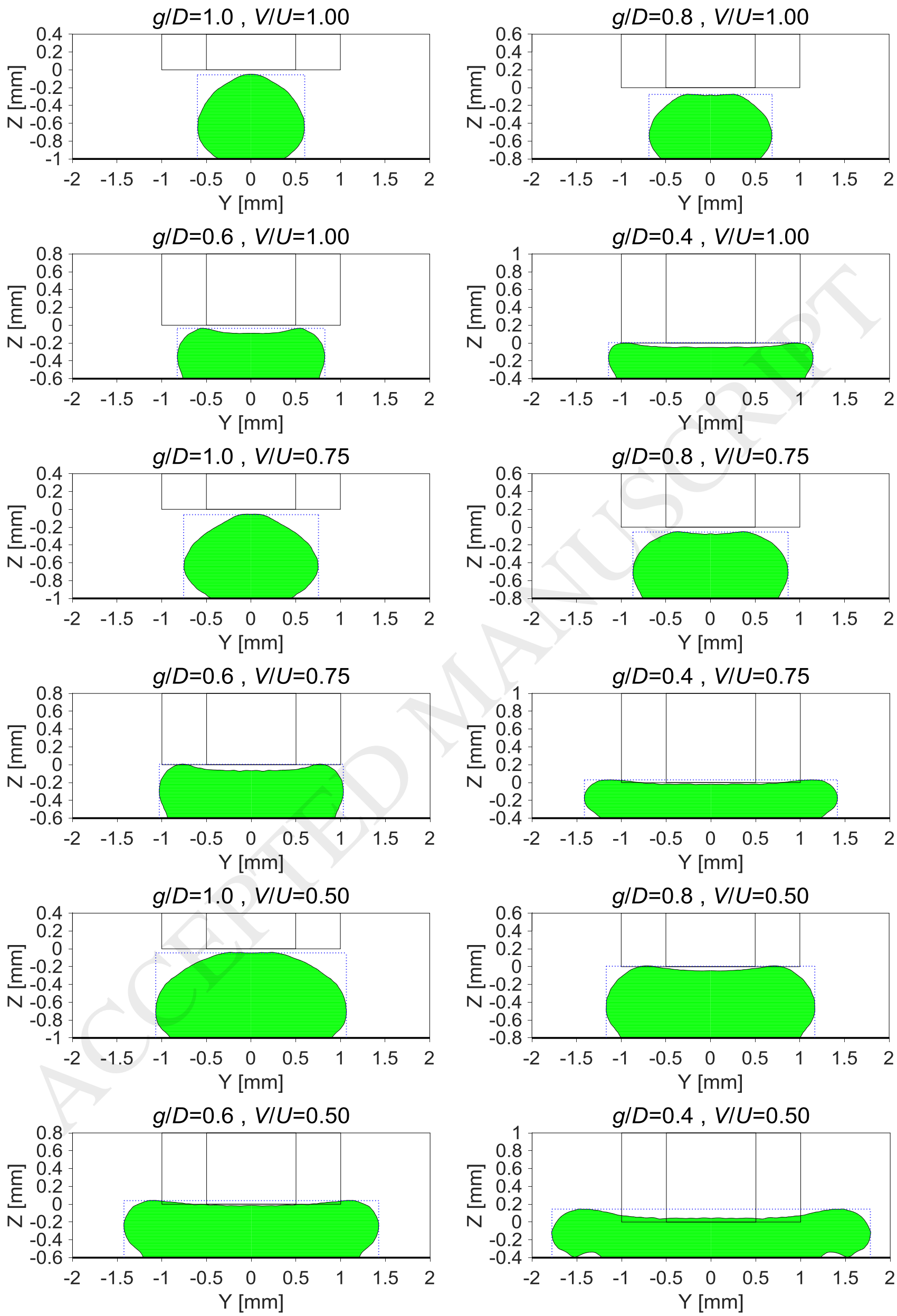

Figure 5. Cross-sections of the strand, for various printing parameters. 

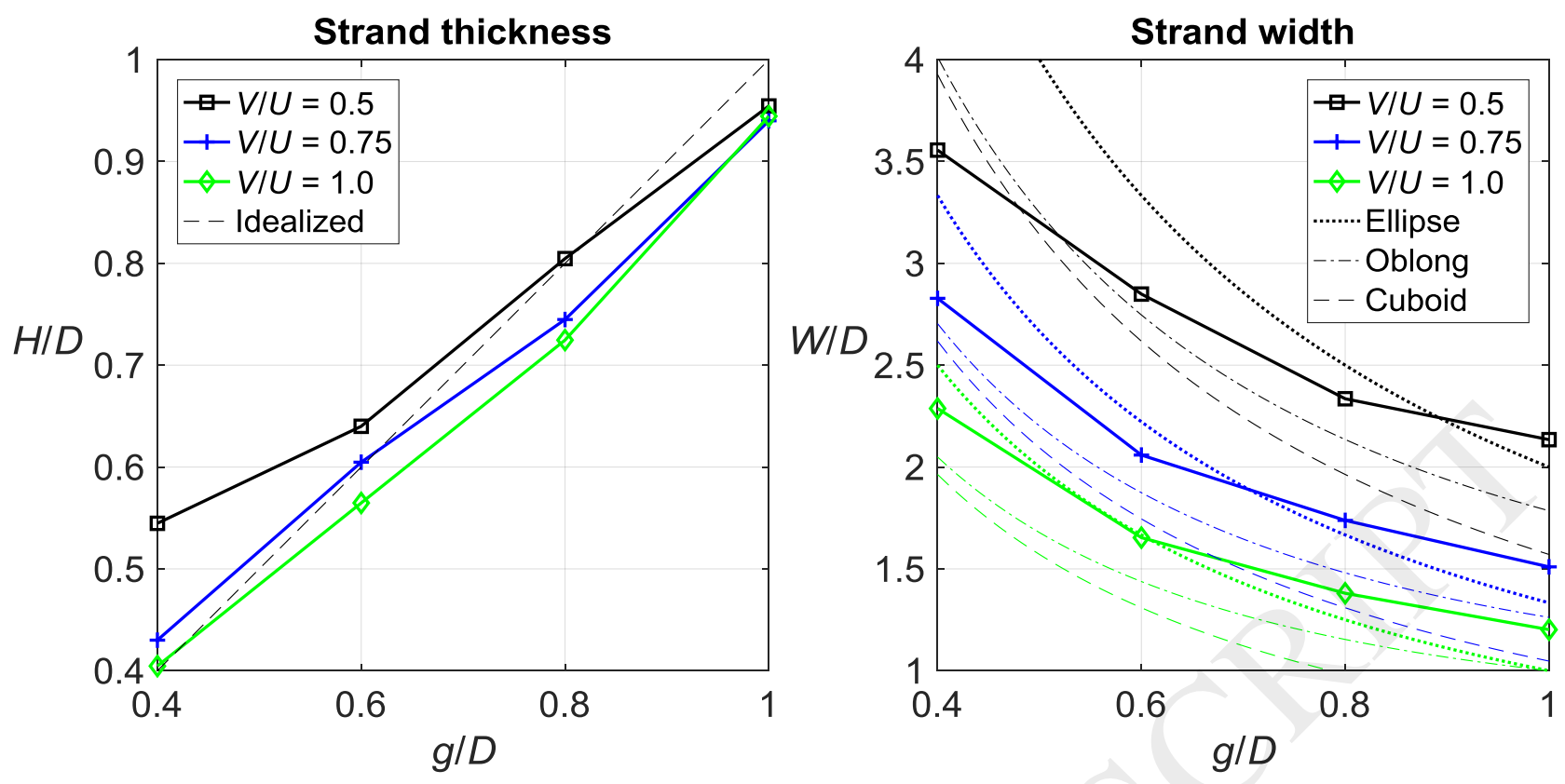

Figure 6. Strand thickness (left) and strand width (right), as a function of the printing parameters. 


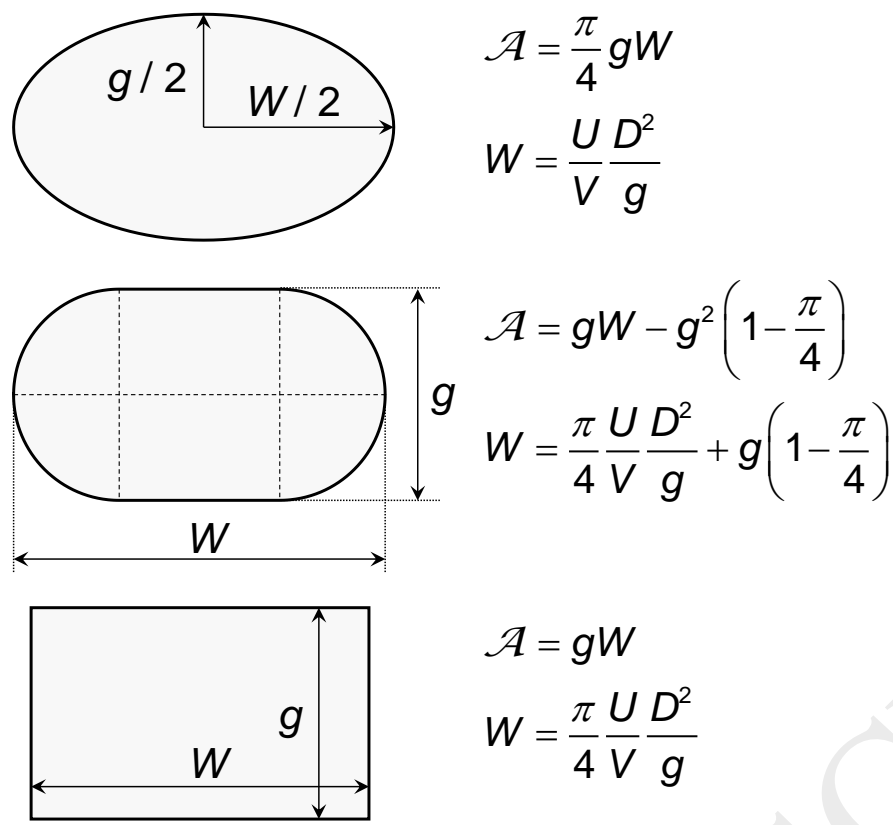

Figure 7. Area and width of idealized cross-sections assuming that the strand thickness equals the gap; from top to bottom: ellipse, oblong and cuboid shapes. 

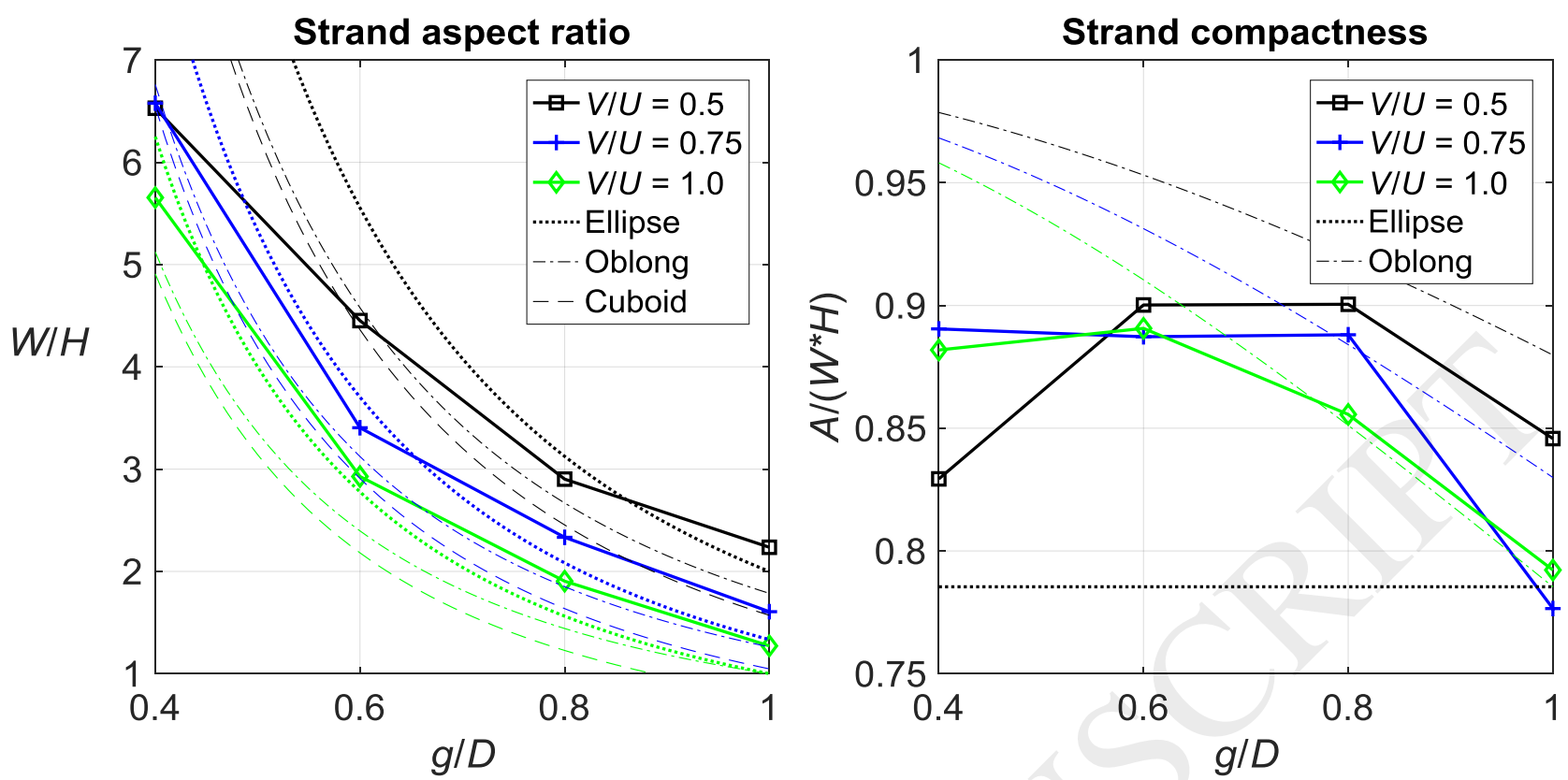

Figure 8. Aspect ratio (left) and compactness (right) of the strand, as a function of the printing parameters. 

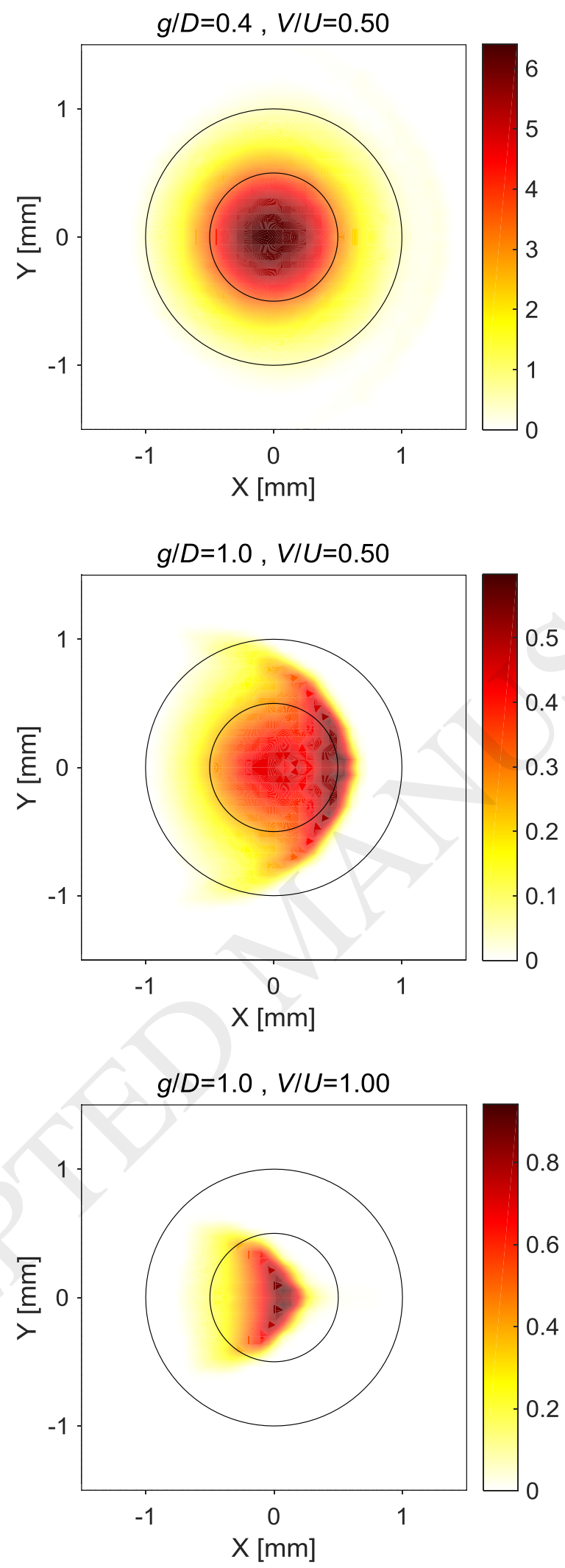

Figure 9. Normalized pressure distribution $\bar{P}$ on the substrate, for different printing conditions. 


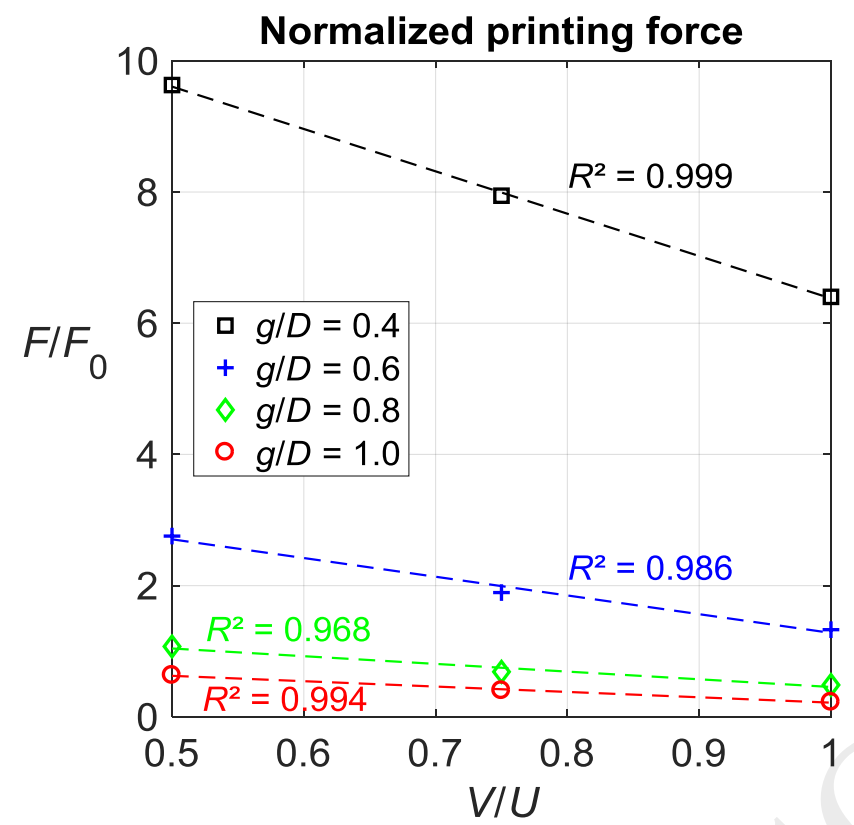

Figure 10. Normalized printing force applied on the substrate, as a function of the printing conditions. 
Table 1. Parameters used in the numerical simulations.

\begin{tabular}{|c|c|c|c|}
\hline Parameters & Nomenclature & Values & Units \\
\hline$D$ & Inner diameter of the nozzle & 1 & $\mathrm{~mm}$ \\
\hline$g$ & Gap distance & $\{0.4,0.6,0.8,1\}$ & $\mathrm{mm}$ \\
\hline$U$ & Average velocity inside the nozzle & 20 & $\mathrm{~mm} / \mathrm{s}$ \\
\hline$V$ & Substrate velocity & $\{10,15,20\}$ & $\mathrm{mm} / \mathrm{s}$ \\
\hline$\rho$ & Density of the molten material & 0.8 & $\mathrm{~g} / \mathrm{cm}^{3}$ \\
\hline$\mu$ & Viscosity of the molten material & 1000 & $\mathrm{~Pa} \cdot \mathrm{s}$ \\
\hline$g_{0}$ & Standard gravity acceleration & -9.81 & $\mathrm{~m} / \mathrm{s}^{2}$ \\
\hline
\end{tabular}

